LINEAR AND NON-LINEAR THEORY OF

GENERALIZED FUNCTIONS AND ITS APPLICATIONS

BANACH CENTER PUBLICATIONS, VOLUME 88

INSTITUTE OF MATHEMATICS

POLISH ACADEMY OF SCIENCES

WARSZAWA 2010

\title{
SINGULAR SOLUTIONS \\ TO SYSTEMS OF CONSERVATION LAWS \\ AND THEIR ALGEBRAIC ASPECTS
}

\author{
V. M. SHELKOVICH \\ Department of Mathematics \\ St.-Petersburg State Architecture and Civil Engineering University \\ 2 Krasnoarmeiskaya 4, 190005, St. Petersburg, Russia \\ E-mail: shelkv@vs1567.spb.edu
}

\begin{abstract}
We discuss the definitions of singular solutions (in the form of integral identities) to systems of conservation laws such as shocks, $\delta$-, $\delta^{\prime}$-, and $\delta^{(n)}$-shocks $(n=2,3, \ldots)$. Using these definitions, the Rankine-Hugoniot conditions for $\delta$ - and $\delta^{\prime}$-shocks are derived. The weak asymptotics method for the solution of the Cauchy problems admitting $\delta$ - and $\delta^{\prime}$-shocks is briefly described. The algebraic aspects of such singular solutions are studied. Namely, explicit formulas for flux-functions of singular solutions are computed. Though the flux-functions are nonlinear, they can be considered as "right" singular superpositions of distributions, thus being well defined Schwartzian distributions. Therefore, singular solutions of Cauchy problems generate algebraic relations between their distributional components.
\end{abstract}

\section{Introduction}

1.1. $\delta^{(n)}$-Shock wave type solutions, $n=0,1,2, \ldots$ There are "nonclassical" situations when, in contrast to Lax's and Glimm's results, the Cauchy problem for a system of conservation laws does not possess a weak $L^{\infty}$-solution except for some particular initial data. In order to solve the Cauchy problem in this "nonclassical" situation, it is necessary to consider the Cauchy problem in a class of singular solutions called $\delta$-shocks and $\delta^{(n)}$-shocks, $n=1,2, \ldots$ Roughly speaking, the $\delta^{(n)}$-shock $(n=0,1,2, \ldots)$ is a solution such that its components contain delta functions and their derivatives up to $n$-th order (for the exact structure of $\delta^{\prime}$ - and $\delta^{\prime}$-shocks see (6) and (9) below).

The theory of $\delta$-shocks has been intensively developed in the last ten years (see [1], [4], 5], 8], 10], [14, [15] and the references therein). One of the typical one-dimensional

2000 Mathematics Subject Classification: Primary 35L65; Secondary 35L67, 76L05.

Key words and phrases: $\delta$ - and $\delta^{\prime}$-shock, the Rankine-Hugoniot conditions, flux-functions singularities, singular superpositions (products) of distributions.

The paper is in final form and no version of it will be published elsewhere. 
system of conservation laws admitting $\delta$-shocks is

$$
L_{1}[u, v]=u_{t}+(F(u, v))_{x}=0, \quad L_{2}[u, v]=v_{t}+(G(u, v))_{x}=0,
$$

where $F(u, v), G(u, v)$ are smooth functions, linear with respect to $v ; u=u(x, t), v=$ $v(x, t) \in \mathbb{R} ; \quad x \in \mathbb{R}$. A particular case of system (1)

$$
u_{t}+(f(u))_{x}=0, \quad v_{t}+(g(u) v)_{x}=0,
$$

(here $F(u, v)=f(u), G(u, v)=v g(u))$ was studied in [4], [5], 10]. $\delta$-Shock in the wellknown Keyfitz-Kranzer system

$$
u_{t}+\left(u^{2}-v\right)_{x}=0, \quad v_{t}+\left(\frac{1}{3} u^{3}-u\right)_{x}=0
$$

(here $F(u, v)=u^{2}-v, G(u, v)=\frac{1}{3} u^{3}-u$ ) was studied in [8]. In [8] $\delta$-shock approximating solutions were constructed, however the notion of an exact singular solution was not defined. Later, in [14, it was first proved that system (3) admits an exact $\delta$-shock solution (6) in the sense of Definition 2.1. In [14], 15] (see also [1]), an exact $\delta$-shock solution to the Keyfitz-Kranzer type system

$$
u_{t}+(f(u)-v)_{x}=0, \quad v_{t}+(g(u))_{x}=0,
$$

was constructed, where $f(u)$ and $g(u)$ are polynomials of degree $n$ and $n+1$, respectively, $n$ is even (here $F(u, v)=f(u)-v, G(u, v)=g(u))$.

Several approaches to solving $\delta$-shock problems are known (for details, see the above cited papers and the references therein). One of them was proposed in [3]- 5], [14, [15]. In these papers the weak asymptotics method was developed for studying the dynamics of propagation and interaction of different singularities of quasi-linear differential equations and systems of conservation laws. Using this method, in [1], 4], [5], 14, [15, for some cases of system (1) with the initial data

$$
u^{0}(x)=u_{+}^{0}(x)+\left[u^{0}(x)\right] H(-x), \quad v^{0}(x)=v_{+}^{0}(x)+\left[v^{0}(x)\right] H(-x)+e^{0} \delta(-x),
$$

the Cauchy problems were solved, where $u_{ \pm}^{0}, v_{ \pm}^{0}$ are given smooth functions, $e^{0}$ is a given constant, $\left[u^{0}\right]=u_{-}^{0}-u_{+}^{0},\left[v^{0}\right]=v_{-}^{0}-v_{+}^{0}$, and $H(x)$ is the Heaviside function, $\delta(x)$ is the delta function. According to [1, 4], [5], 14], 15], the $\delta$-shock wave type solutions of these Cauchy problems have the form

$$
\begin{aligned}
& u(x, t)=u_{+}(x, t)+[u(x, t)] H(-x+\phi(t)), \\
& v(x, t)=v_{+}(x, t)+[v(x, t)] H(-x+\phi(t))+e(t) \delta(-x+\phi(t)),
\end{aligned}
$$

where $u_{ \pm}, v_{ \pm}, e(t), \phi(t)$ are desired functions, $[u]=u_{-}-u_{+},[v]=v_{-}-v_{+}, x=\phi(t)$ is the discontinuity curve.

In [13], a concept of $\delta^{(n)}$-shock wave type solutions was introduced and the theory of $\delta^{\prime}$-shocks was established for the one-dimensional system of conservation laws

$$
\begin{aligned}
L_{1}[u] & =u_{t}+(f(u))_{x}=0, \\
L_{2}[u, v] & =v_{t}+\left(f^{\prime}(u) v\right)_{x}=0, \\
L_{3}[u, v, w] & =w_{t}+\left(f^{\prime \prime}(u) v^{2}+f^{\prime}(u) w\right)_{x}=0,
\end{aligned}
$$

where $f(u)$ is a smooth function. In [13, in the framework of the weak asymptotics method for a particular case of system (7), when $f(u)=u^{2}$, the Cauchy problem with the singular 
initial data

$$
\begin{aligned}
u^{0}(x) & =u_{+}^{0}(x)+\left[u^{0}(x)\right] H(-x), \\
v^{0}(x) & =v_{+}^{0}(x)+\left[v^{0}(x)\right] H(-x)+e^{0} \delta(-x), \\
w^{0}(x) & =w_{+}^{0}(x)+\left[w^{0}(x)\right] H(-x)+g^{0} \delta(-x)+h^{0} \delta^{\prime}(-x),
\end{aligned}
$$

was constructed, where $u_{ \pm}^{0}(x), v_{ \pm}^{0}(x), w_{ \pm}^{0}(x)$, are given smooth functions; $e^{0}, g^{0}, h^{0}$ are given constants, $\delta^{\prime}(x)$ is the derivative of the delta function. This solution has the form

$$
\begin{aligned}
& u(x, t)=u_{+}(x, t)+[u(x, t)] H(-x+\phi(t)), \\
& v(x, t)=v_{+}(x, t)+[v(x, t)] H(-x+\phi(t))+e(t) \delta(-x+\phi(t)), \\
& w(x, t)=w_{+}(x, t)+[w(x, t)] H(-x+\phi(t))+g(t) \delta(-x+\phi(t)) \\
&+h(t) \delta^{\prime}(-x+\phi(t))
\end{aligned}
$$

where $u_{ \pm}(x, t), v_{ \pm}(x, t), w_{ \pm}(x, t), \phi(t), e(t), g(t), h(t)$ are the desired functions. In [16], for a particular case of system (7), when $f(u)=u^{2}$, the Riemann problem admitting $\delta$-, $\delta^{\prime}$-shock wave type solutions and vacuum states was solved.

$\delta^{(n)}$-Shocks $(n=0,1,2, \ldots)$ do not satisfy the standard $L^{\infty}$-integral identities, and to deal with them we need:

- to discover a proper notion of a singular solution, i.e., to define in which sense it may satisfy a nonlinear system;

- to devise some way to define singular superpositions (products) of distributions (for example, a product of the Heaviside function and the delta function).

Fortunately, it appears that the above-mentioned weak asymptotics method is a proper technique to deal with $\delta$ - and $\delta^{\prime}$-shocks.

1.2. Main results. In Sec. 2. Definition 2.1 of $\delta$-shocks for the system of conservation law (1) is introduced, and the Rankine-Hugoniot conditions (16) are derived. In Sec. 3 . Definition 3.1 of $\delta^{\prime}$-shock for system (7) is introduced, and the Rankine-Hugoniot conditions (21)-24) are derived. In Sec. 4. we briefly describe the weak asymptotics method for the solution of the Cauchy problems admitting $\delta$ - and $\delta^{\prime}$-shocks.

In Subsec. 5.2, the algebraic aspects of $\delta$ - and $\delta^{\prime}$-shocks in systems (1), (7) are studied. It is well known that in the general case the product of distributions either is not a Schwartz distribution or it is a Schwartz distribution not uniquely defined. Nevertheless, we show that singular solutions of the Cauchy problems generate algebraic relations between their distributional components. More precisely, one can calculate flux-functions of $\delta$ - and $\delta^{\prime}$-shock solutions (see Theorems 5.1, 5.2. Though flux-functions are nonlinear, they can be considered as "right" singular superpositions of distributions and are well defined Schwartzian distributions. Thus a "right" singular superposition is determined only in the context of solving the Cauchy problem. Note that in our paper [9] the flux-functions of $\delta$-shocks for system (1) with piecewise constant initial data were calculated. In Subsec. 5.3, we discuss "strange" specific properties of the "right" singular superpositions. We also discuss the possibilities to use the nonconservative product [10], [2] and the Colombeau theory to construct a $\delta$-shock for system (3), (4) and a $\delta^{\prime}$-shock for system (7). 
2. $\delta$-Shock type solutions and the Rankine-Hugoniot conditions. Suppose that $\Gamma=\left\{\gamma_{i}: i \in I\right\}$ is a graph in the upper half-plane $\{(x, t): x \in \mathbb{R}, t \in[0, \infty)\} \in \mathbb{R}^{2}$ containing smooth $\operatorname{arcs} \gamma_{i}=\left\{(x, t): S_{i}(x, t)=0\right\}, S_{i} \in C^{1}, S_{i x} \neq 0, \quad i \in I$, and $I$ is a finite set. By $I_{0}$ we denote a subset of $I$ such that an arc $\gamma_{k}$ for $k \in I_{0}$ starts from points of the $x$-axis. Denote by $\Gamma_{0}=\left\{x_{k}^{0}: k \in I_{0}\right\}$ the set of initial points of arcs $\gamma_{k}, k \in I_{0}$. Here arcs of a graph have the orientation corresponding to increasing time $t$. Consider the $\delta$-shock type initial data

$$
\left(u^{0}(x), v^{0}(x)\right), \quad \text { where } \quad v^{0}(x)=\widehat{v}^{0}(x)+e^{0} \delta\left(\Gamma_{0}\right),
$$

$u^{0}, \widehat{v}^{0} \in L^{\infty}(\mathbb{R} ; \mathbb{R}), \quad e^{0} \delta\left(\Gamma_{0}\right) \stackrel{\text { def }}{=} \sum_{k \in I_{0}} e_{k}^{0} \delta\left(x-x_{k}^{0}\right), \quad e_{k}^{0}$ are constants, $k \in I_{0}$.

Definition $2.1([4,[5])$. A pair of distributions $(u, v)$ and a graph $\Gamma$, where $v(x, t)$ has the form of the sum

$$
v(x, t)=\widehat{v}(x, t)+e(x, t) \delta(\Gamma),
$$

$u, \widehat{v} \in L^{\infty}(\mathbb{R} \times(0, \infty) ; \mathbb{R}), \quad e(x, t) \delta(\Gamma) \stackrel{\text { def }}{=} \sum_{i \in I} e_{i}(x, t) \delta\left(\gamma_{i}\right), \quad e_{i}(x, t) \in C(\Gamma), i \in I$, is called a $\delta$-shock wave type solution of the Cauchy problem (1), (10) if the integral identities

$$
\begin{array}{r}
\int_{0}^{\infty} \int\left(u \varphi_{t}+F(u, \widehat{v}) \varphi_{x}\right) d x d t+\int u^{0}(x) \varphi(x, 0) d x=0 \\
\int_{0}^{\infty} \int\left(\widehat{v} \varphi_{t}+G(u, \widehat{v}) \varphi_{x}\right) d x d t+\sum_{i \in I} \int_{\gamma_{i}} e_{i}(x, t) \frac{\delta \varphi(x, t)}{\delta t} \frac{d l}{\sqrt{1+u_{\delta}^{2}}} \\
+\int \widehat{v}^{0}(x) \varphi(x, 0) d x+\sum_{k \in I_{0}} e_{k}^{0} \varphi\left(x_{k}^{0}, 0\right)=0
\end{array}
$$

hold for all test functions $\varphi(x, t) \in \mathcal{D}(\mathbb{R} \times[0, \infty))$, where

$$
\left.\frac{\delta \varphi}{\delta t}\right|_{\gamma_{i}}=\left.\left(\frac{\partial \varphi}{\partial t}-\frac{S_{i t}}{S_{i x}} \frac{\partial \varphi}{\partial x}\right)\right|_{S_{i}(x, t)=0}
$$

is a $\delta$-derivative with respect to time [7, 5.2.(15)], which is the tangential derivative on the graph $\gamma_{i}$;

$$
\left.u_{\delta}(x, t)\right|_{\gamma_{i}}=-\left.\frac{S_{i t}}{S_{i x}}\right|_{\gamma_{i}}, \quad i \in I,
$$

is the velocity of a $\delta$-shock on $\gamma_{i} ; \int_{\gamma_{i}} \cdot d l$ is the line integral over the arc $\gamma_{i}$. Here the delta function $\delta\left(\gamma_{i}\right)$ on the curve $\gamma_{i}$ is defined as in [7, 5.3].

The integral identities 11 differ from the standard $L^{\infty}$-integral identities by the additional terms $\sum_{i \in I} \int_{\gamma_{i}} e_{i}(x, t) \frac{\delta \varphi(x, t)}{\delta t} \frac{d l}{\sqrt{1+u_{\delta}^{2}}}$ in the second identity which appear due to the delta function in $v$.

If arcs of the graph $\Gamma=\left\{\gamma_{i}: i \in I\right\}$ have the form $\gamma_{i}=\left\{(x, t): x=\phi_{i}\right\}, \quad \phi_{i}(t) \in$ $C^{1}(0,+\infty), i \in I$, then

$$
\left.\frac{\delta \varphi}{\delta t}\right|_{\gamma_{i}}=\left.\sqrt{1+\left(\dot{\phi}_{i}(t)\right)^{2}} \frac{\partial \varphi(x, t)}{\partial \mathbf{l}}\right|_{\gamma_{i}}=\frac{d \varphi\left(\phi_{i}(t), t\right)}{d t},
$$


where $\frac{\partial \varphi}{\partial \mathbf{l}}$ is the tangential derivative on the graph $\gamma_{i}$ along the unit vector $\mathbf{l}=\left(-\nu_{2}, \nu_{1}\right)=$ $\frac{\left(\dot{\phi}_{i}(t), 1\right)}{\sqrt{1+\left(\dot{\phi}_{i}(t)\right)^{2}}}, \mathbf{n}=\left(\nu_{1}, \nu_{2}\right)=\frac{\left(1,-\dot{\phi}_{i}(t)\right)}{\sqrt{1+\left(\dot{\phi}_{i}(t)\right)^{2}}}$ is the unit oriented normal to $\gamma_{i}$.

Now, by using Definition 2.1, we derive the $\delta$-shock Rankine-Hugoniot conditions for system (1).

THEOREM 2.2 ([1], 15]). Let us assume that $\Omega \subset \mathbb{R} \times(0, \infty)$ is a region cut by a smooth curve $\Gamma=\{(x, t): S(x, t)=0\}$ into the left-and right-hand parts $\Omega_{\mp}$. Let $(u, v)$, $\Gamma$ be a $\delta$-shock wave type solution of system (1), and suppose that $u, v$ are smooth in $\Omega_{ \pm}$and have one-sided limits $u_{ \pm}, v_{ \pm}$on $\Gamma$. Then the Rankine-Hugoniot conditions for the $\delta$-shock

$$
u_{\delta}=\left.\frac{[F(u, v)}{[u]}\right|_{\Gamma},\left.\quad \frac{\delta e(x, t)}{\delta t}\right|_{\Gamma}=\left([G(u, v)]_{\Gamma}-[v]_{\Gamma} u_{\delta}\right) \frac{S_{x}}{\left|S_{x}\right|},
$$

where $u_{\delta}(x, t)$ is the velocity (13) of a $\delta$-shock, $[a(u, v)]=a\left(u_{-}, v_{-}\right)-a\left(u_{+}, v_{+}\right)$is, as usual, the jump of the function $a(u(x, t), v(x, t))$ across the discontinuity curve $\Gamma$.

If $\Gamma=\{(x, t): x=\phi(t)\}, \phi(t) \in C^{1}(0,+\infty)$, then 15 reads

$$
\dot{\phi}(t)=\left.\frac{[F(u, v)]}{[u]}\right|_{x=\phi(t)}, \quad \dot{e}(t)=\left.\left([G(u, v)]-[v] \frac{[F(u, v)]}{[u]}\right)\right|_{x=\phi(t)},
$$

where $e$ can be treated as a function of the single variable $t$, so that $e(t) \stackrel{\text { def }}{=} e(\phi(t), t)$.

Proof. Let $\mathbf{n}=\left(\nu_{1}, \nu_{2}\right)=\frac{\left(S_{x}, S_{t}\right)}{\left|\nabla_{(x, t)}\right|}$ be the unit normal to the curve $\Gamma$ oriented from $\Omega_{-}$ to $\Omega_{+}, \quad \mathbf{l}=\left(-\nu_{2}, \nu_{1}\right)=\frac{\left(-S_{t}, S_{x}\right)}{\left|\nabla_{(x, t)}\right|}$ be a unit tangential vector to $\Gamma, \nabla_{(x, t)} S_{i}=\left(S_{i x}, S_{i t}\right)$.

For any test function $\varphi \in \mathcal{D}(\Omega)$ we have $\varphi(x, t)=0$, if $(x, t) \notin G, \bar{G} \subset \Omega$. Selecting $\varphi(x, t)$ with compact support in $\Omega_{ \pm}$, we deduce from (11) that (1) holds in $\Omega_{ \pm}$, respectively. Now, choosing $\varphi(x, t)$ with support in $\Omega$, we deduce from the first identity of (11) that

$$
\begin{aligned}
\int_{0}^{\infty} \int\left(u \varphi_{t}\right. & \left.+F(u, \widehat{v}) \varphi_{x}\right) d x d t \\
& =\iint_{\Omega_{-} \cap G}\left(u \varphi_{t}+F(u, \widehat{v}) \varphi_{x}\right) d x d t+\iint_{\Omega_{+} \cap G}\left(u \varphi_{t}+F(u, \widehat{v}) \varphi_{x}\right) d x d t
\end{aligned}
$$

Since $u_{t}+(F(u, v))_{x}=0$ for $(x, t) \in \Omega_{ \pm}$, integrating by parts, we obtain

$$
\begin{aligned}
\iint_{\Omega_{ \pm} \cap G}\left(u \varphi_{t}+F(u, \widehat{v})\right. & \left.\varphi_{x}\right) d x d t \\
& =\mp \int_{\Gamma}\left(\nu_{2} u_{ \pm}+\nu_{1} F\left(u_{ \pm}, v_{ \pm}\right)\right) \varphi d l-\int_{\Omega_{ \pm} \cap G \cap \mathbb{R}} u^{0}(x) \varphi(x, 0) d x .
\end{aligned}
$$

Adding the latter relations, we have

$$
\begin{aligned}
\int_{0}^{\infty} \int\left(u \varphi_{t}+F(u, \widehat{v}) \varphi_{x}\right) d x d t+\int u^{0}(x) \varphi(x, 0) d x & \\
& =\int_{\Gamma}\left([F(u, v)] \nu_{1}+[u] \nu_{2}\right) \varphi(x, t) d l=0
\end{aligned}
$$


for all $\varphi(x, t) \in \mathcal{D}(\Omega)$. This implies the first relation in 15 . In the same way we obtain

$$
\begin{aligned}
\int_{0}^{\infty} \int\left(\widehat{v} \varphi_{t}+G(u, \widehat{v}) \varphi_{x}\right) d x d t+\int \widehat{v}^{0}(x) \varphi(x, 0) d x & \\
& =\int_{\Gamma}\left([G(u, v)] \nu_{1}+[v] \nu_{2}\right) \varphi(x, t) d l .
\end{aligned}
$$

Next, integrating by parts, it is easy to see that

$$
\begin{aligned}
& \sum_{i \in I} \int_{\gamma_{i}} e_{i}(x, t) \frac{\delta \varphi(x, t)}{\delta t} \frac{d l}{\sqrt{1+u_{\delta}^{2}}} \\
&=-\sum_{i \in I} \int_{\gamma_{i}} \frac{\delta e_{i}(x, t)}{\delta t} \varphi(x, t) \frac{d l}{\sqrt{1+u_{\delta}^{2}}}-\left.\sum_{k \in I_{0}} e^{0} \varphi(x, 0)\right|_{S_{k}(x, 0)=0},
\end{aligned}
$$

where the $\delta$-derivative $\frac{\delta \varphi}{\delta t}$ is defined in $(12)$. Adding (17) and 18 , we obtain

$$
\int_{\Gamma}\left([G(u, v)] \nu_{1}+[v] \nu_{2}-\frac{\delta e(x, t)}{\delta t} \frac{1}{\sqrt{1+u_{\delta}^{2}}}\right) \varphi(x, t) d l=0, \quad \forall \varphi(x, t) \in \mathcal{D}(\Omega) .
$$

Thus the second relation in (15) holds.

If $\Gamma=\{(x, t): x=\phi(t)\}, \phi(t) \in C^{1}(0,+\infty)$, in view of (14), condition 15) can be rewritten as 16 .

The first equation in (15) (or $\sqrt{16}$ ) is the standard Rankine-Hugoniot condition for shock. The left-hand side of the second equation in (15) (or the right-hand side of the second equation in (16)) is called the Rankine-Hugoniot deficit in $v$.

3. $\delta^{\prime}$-Shock type solutions and the Rankine-Hugoniot conditions. Denote by $\widetilde{\mathcal{C}}(\mathbb{R} \times(0, \infty) ; \mathbb{R})$ the class of piecewise-smooth functions. Let $\Gamma=\left\{\gamma_{i}: i \in I\right\}$ be the graph introduced in Sec. 2. The initial data

$$
\begin{aligned}
\left(u^{0}(x), v^{0}(x), w^{0}(x)\right), \quad \text { where } & v^{0}(x)=\widehat{v}^{0}(x)+e^{0} \delta\left(\Gamma_{0}\right) \\
& w^{0}(x)=\widehat{w}^{0}(x)+g^{0} \delta\left(\Gamma_{0}\right)+h^{0} \delta^{\prime}\left(\Gamma_{0}\right),
\end{aligned}
$$

and $u^{0}, \widehat{v}^{0}, \widehat{w}^{0} \in \widetilde{\mathcal{C}}(\mathbb{R} ; \mathbb{R})$, will be called $\delta^{\prime}$-shock type initial data. Here, by definition,

$$
\begin{gathered}
e^{0} \delta\left(\Gamma_{0}\right) \stackrel{\text { def }}{=} \sum_{k \in I_{0}} e_{k}^{0} \delta\left(x-x_{k}^{0}\right), \\
g^{0} \delta\left(\Gamma_{0}\right) \stackrel{\text { def }}{=} \sum_{k \in I_{0}} g_{k}^{0} \delta\left(x-x_{k}^{0}\right), \\
h^{0} \delta\left(\Gamma_{0}\right) \stackrel{\text { def }}{=} \sum_{k \in I_{0}} h_{k}^{0} \delta^{\prime}\left(x-x_{k}^{0}\right),
\end{gathered}
$$

where $e_{k}^{0}, g_{k}^{0}, h_{k}^{0}$ are constants, $k \in I_{0}$.

Definition 3.1 ([13]). A triple of distributions $(u, v, w)$ and a graph $\Gamma$, where $v(x, t)$ and $w(x, t)$ have the form of the sums

$$
v(x, t)=\widehat{v}(x, t)+e(x, t) \delta(\Gamma), \quad w(x, t)=\widehat{w}(x, t)+g(x, t) \delta(\Gamma)+h(x, t) \delta^{\prime}(\Gamma),
$$


and $u, \widehat{v}, \widehat{w} \in \widetilde{\mathcal{C}}(\mathbb{R} \times(0, \infty) ; \mathbb{R})$,

$$
\begin{aligned}
& e \delta(\Gamma) \stackrel{\text { def }}{=} \sum_{i \in I} e_{i} \delta\left(\gamma_{i}\right), \\
& g \delta(\Gamma) \stackrel{\text { def }}{=} \sum_{i \in I} g_{i} \delta\left(\gamma_{i}\right), \\
& h \delta^{\prime}(\Gamma) \stackrel{\text { def }}{=} \sum_{i \in I} h_{i}(x, t) \delta^{\prime}\left(\gamma_{i}\right),
\end{aligned}
$$

where $e_{i}(x, t), g_{i}(x, t), h_{i}(x, t) \in C^{1}(\Gamma), i \in I$, is called a $\delta^{\prime}$-shock wave type solution of the Cauchy problem (7), 19) if the integral identities

$$
\begin{array}{r}
\int_{0}^{\infty} \int\left(u \varphi_{t}+f(u) \varphi_{x}\right) d x d t+\int u^{0}(x) \varphi(x, 0) d x=0, \\
\int_{0}^{\infty} \int \widehat{v}\left(\varphi_{t}+f^{\prime}(u) \varphi_{x}\right) d x d t+\sum_{i \in I} \int_{\gamma_{i}} e_{i}(x, t) \frac{\delta \varphi(x, t)}{\delta t} \frac{d l}{\sqrt{1+u_{\delta}^{2}}} \\
+\int \widehat{v}^{0}(x) \varphi(x, 0) d x+\sum_{k \in I_{0}} e_{k}^{0} \varphi\left(x_{k}^{0}, 0\right)=0 \\
+\sum_{i \in I}\left(\int_{\gamma_{i}} g_{i}(x, t) \frac{\delta \varphi(x, t)}{\delta t} \frac{d l}{\sqrt{1+u_{\delta}^{2}}}+\int_{\gamma_{i}} h_{i}(x, t) \frac{\delta \varphi_{x}(x, t)}{\delta t} \frac{d l}{\sqrt{1+u_{\delta}^{2}}}\right. \\
\left.+\int_{\gamma_{i}} \frac{\delta e_{i}^{2}(x, t)}{\delta t}-h_{i}(x, t) \frac{\delta[u(x, t)]}{\delta t} \varphi_{x}(x, t) \frac{d l}{\sqrt{1+u_{\delta}^{2}}}\right) \\
\left.+\int(x, t)\right] \widehat{w}^{\prime}(x) \varphi(x, 0) d x+\sum_{k \in I_{0}} g_{k}^{0} \varphi\left(x_{k}^{0}, 0\right)+\sum_{k \in I_{0}} h_{k}^{0} \varphi_{x}\left(x_{k}^{0}, 0\right)=0
\end{array}
$$

hold for all $\varphi(x, t) \in \mathcal{D}(\mathbb{R} \times[0, \infty))$. The derivative of the delta function $\delta^{\prime}\left(\gamma_{i}\right)$ on the curve $\gamma_{i}$ is defined in [7, 5.3; 5.5].

The integral identities (20) differ from the standard $L^{\infty}$-integral identities by the additional terms in the second and third identities. The terms

$$
\sum_{i \in I} \int_{\gamma_{i}} e_{i}(x, t) \frac{\delta \varphi(x, t)}{\delta t} \frac{d l}{\sqrt{1+u_{\delta}^{2}}}, \quad \sum_{i \in I} \int_{\gamma_{i}} g_{i}(x, t) \frac{\delta \varphi(x, t)}{\delta t} \frac{d l}{\sqrt{1+u_{\delta}^{2}}}
$$

appear due to the delta functions in $v w$, and the terms

$$
\sum_{i \in I}\left(\int_{\gamma_{i}} h_{i}(x, t) \frac{\delta \varphi_{x}(x, t)}{\delta t} \frac{d l}{\sqrt{1+u_{\delta}^{2}}}+\int_{\gamma_{i}} \frac{\frac{\delta e_{i}^{2}(x, t)}{\delta t}-h_{i}(x, t) \frac{\delta[u(x, t)]}{\delta t}}{[u(x, t)]} \varphi_{x}(x, t) \frac{d l}{\sqrt{1+u_{\delta}^{2}}}\right)
$$

appear due to the derivative of the delta function in $w$.

THEOREM $3.2([13])$. Let us assume that $\Omega \subset \mathbb{R} \times[0, \infty)$ is some region cut by a curve $\Gamma=\{(x, t): x=\phi(t)\}, \phi(t) \in C^{1}(0,+\infty)$ into left- and right-hand parts $\Omega_{ \pm}=\{(x, t) \in$ $\Omega: \pm(x-\phi(t))>0\}$. Let $(u(x, t), v(x, t), w(x, t)), \Gamma$ be a generalized $\delta^{\prime}$-shock wave type 
solution of system (7). Assume that $(u, v, w)$ are smooth in the domains $\Omega_{ \pm}$and have one-sided limits $u_{ \pm}, v_{ \pm}, w_{ \pm}$on $\Gamma$, which are supposed to be continuous functions on $\Gamma$. Then the Rankine-Hugoniot conditions for the $\delta^{\prime}$-shock

$$
\begin{aligned}
\dot{\phi}(t) & =\left.\frac{[f(u)]}{[u]}\right|_{x=\phi(t)}, \\
\dot{e}(t) & =\left.\left(\left[f^{\prime}(u) v\right]-[v] \frac{[f(u)]}{[u]}\right)\right|_{x=\phi(t)}, \\
\dot{g}(t) & =\left.\left(\left[f^{\prime \prime}(u) v^{2}+f^{\prime}(u) w\right]-[w] \frac{[f(u)]}{[u]}\right)\right|_{x=\phi(t)}, \\
\frac{d}{d t}(h(t)[u(\phi(t), t)]) & =\frac{d e^{2}(t)}{d t}
\end{aligned}
$$

hold along $\Gamma$. Here $e(t) \stackrel{\text { def }}{=} e(\phi(t), t), g(t) \stackrel{\text { def }}{=} g(\phi(t), t), h(t) \stackrel{\text { def }}{=} h(\phi(t), t)$.

The system of the Rankine-Hugoniot conditions (21)-24) determines the trajectory $x=\phi(t)$ of a $\delta^{\prime}$-shock wave and the coefficients $e(t), g(t), h(t)$ of the singularities. The first equation in this system is the "standard" Rankine-Hugoniot condition for the shock, while the first and second equations are the "standard" Rankine-Hugoniot conditions for the $\delta$-shock (cf. (16)). The right-hand sides of equalities 222, 23) are the first Rankine-Hugoniot deficits, while the right-hand side of (24) is the second RankineHugoniot deficit.

\section{The Cauchy problems admitting $\delta$ - and $\delta^{\prime}$-shocks}

4.1. Weak asymptotic solutions. We are going to introduce a notion of weak asymptotic solution, which is one of the most important in the weak asymptotics method.

Let $\alpha \in \mathbb{R}$. Denote by $O_{\mathcal{D}^{\prime}}\left(\varepsilon^{\alpha}\right), \varepsilon \rightarrow+0$ a collection of distributions (with respect to x) $f(x, t, \varepsilon) \in \mathcal{D}^{\prime}\left(\mathbb{R}_{x}\right), x \in \mathbb{R}, t \in[0, T], \varepsilon>0$ such that

$$
\langle f(\cdot, t, \varepsilon), \psi(\cdot)\rangle=O\left(\varepsilon^{\alpha}\right), \quad \varepsilon \rightarrow+0,
$$

for any test function $\psi(x) \in \mathcal{D}(\mathbb{R}), x \in \mathbb{R}$. Moreover, $\langle f(\cdot, t, \varepsilon), \psi(\cdot)\rangle$ is a continuous function in $t$, and the estimate $O\left(\varepsilon^{\alpha}\right)$ is understood in the standard sense being uniform with respect to $t$ in $[0, T]$. The notation $o_{\mathcal{D}^{\prime}}\left(\varepsilon^{\alpha}\right), \varepsilon \rightarrow+0$ is understood correspondingly.

Definition $4.1\left(\left[4\right.\right.$, [5]). A pair of functions $\left(u_{\varepsilon}(x, t), v_{\varepsilon}(x, t)\right)$ which are smooth as $\varepsilon>0, t \in[0, T]$ is called a weak asymptotic solution of the Cauchy problem (1), 10 if

$$
\begin{aligned}
L_{1}\left[u_{\varepsilon}(x, t), v_{\varepsilon}(x, t)\right] & =o_{\mathcal{D}^{\prime}}(1), \\
L_{2}\left[u_{\varepsilon}(x, t), v_{\varepsilon}(x, t)\right] & =o_{\mathcal{D}^{\prime}}(1), \\
u_{\varepsilon}(x, 0) & =u^{0}(x)+o_{\mathcal{D}^{\prime}}(1), \\
v_{\varepsilon}(x, 0) & =v^{0}(x)+o_{\mathcal{D}^{\prime}}(1), \quad \varepsilon \rightarrow+0,
\end{aligned}
$$

where the first two estimates are uniform in $t \in[0, T]$. 
Definition $4.2(13])$. A triple of functions $\left(u_{\varepsilon}(x, t), v_{\varepsilon}(x, t), w_{\varepsilon}(x, t)\right)$, smooth as $\varepsilon>0$, $t \in[0, T]$ is called a weak asymptotic solution of the Cauchy problem (7), (19) if

$$
\begin{aligned}
L_{1}\left[u_{\varepsilon}(x, t)\right] & =o_{\mathcal{D}^{\prime}}(1), \\
L_{2}\left[u_{\varepsilon}(x, t), v_{\varepsilon}(x, t)\right] & =o_{\mathcal{D}^{\prime}}(1), \\
L_{3}\left[u_{\varepsilon}(x, t), v_{\varepsilon}(x, t), w_{\varepsilon}(x, t)\right] & =o_{\mathcal{D}^{\prime}}(1), \\
u_{\varepsilon}(x, 0) & =u^{0}(x)+o_{\mathcal{D}^{\prime}}(1), \\
v_{\varepsilon}(x, 0) & =v^{0}(x)+o_{\mathcal{D}^{\prime}}(1), \\
w_{\varepsilon}(x, 0) & =w^{0}(x)+o_{\mathcal{D}^{\prime}}(1), \quad \varepsilon \rightarrow+0,
\end{aligned}
$$

where the first three estimates are uniform in $t \in[0, T]$.

In (25) and (26) all distributions in $u, v, w$ depend on $t$ as a parameter.

Recall that one of the methods for studying singular solutions to systems of conservation laws is the vanishing viscosity method which introduces viscosity terms in the right-hand sides of a system of conservation laws. In this case viscosity terms admit estimates of the form $o_{\mathcal{D}^{\prime}}(1)$, and, consequently, a viscosity solution can be considered as a weak asymptotic solution. Thus a viscosity solution is a particular case of a weak asymptotic solution of the Cauchy problem, and our notation $o_{\mathcal{D}^{\prime}}(1)$ in the right-hand sides of the equations (25) and (26) can be interpreted as a small viscosity.

4.2. Solving the Cauchy problems. In the framework of the weak asymptotics method, we find a $\delta$ - or $\delta^{\prime}$-shock wave type solution of the Cauchy problem as the weak limit

$$
u(x, t)=\lim _{\varepsilon \rightarrow+0} u_{\varepsilon}(x, t), \quad v(x, t)=\lim _{\varepsilon \rightarrow+0} v_{\varepsilon}(x, t), \quad w(x, t)=\lim _{\varepsilon \rightarrow+0} w_{\varepsilon}(x, t),
$$

of the weak asymptotic solution $\left(u_{\varepsilon}, v_{\varepsilon}\right)$ or $\left(u_{\varepsilon}, v_{\varepsilon}, w_{\varepsilon}\right)$ to the corresponding Cauchy problem.

Let $\left(u_{\varepsilon}, v_{\varepsilon}\right)$ be a weak asymptotic solution of the Cauchy problem (1), (10), and $\left(u_{\varepsilon}, v_{\varepsilon}, w_{\varepsilon}\right)$ be a weak asymptotic solution of the Cauchy problem (7), 19). According to 25 and $(26)$, for all $\varphi(x, t) \in \mathcal{D}(\mathbb{R} \times[0, \infty))$ we have

$$
\begin{aligned}
& \lim _{\varepsilon \rightarrow+0} \int_{0}^{\infty} \int L_{1}\left[u_{\varepsilon}(x, t), v_{\varepsilon}(x, t)\right] \varphi(x, t) d x d t=0, \\
& \lim _{\varepsilon \rightarrow+0} \int_{0}^{\infty} \int L_{2}\left[u_{\varepsilon}(x, t), v_{\varepsilon}(x, t)\right] \varphi(x, t) d x d t=0
\end{aligned}
$$

and

$$
\begin{aligned}
& \lim _{\varepsilon \rightarrow+0} \int_{0}^{\infty} \int L_{1}\left[u_{\varepsilon}(x, t)\right] \varphi(x, t) d x d t=0, \\
& \lim _{\varepsilon \rightarrow+0} \int_{0}^{\infty} \int L_{2}\left[u_{\varepsilon}(x, t), v_{\varepsilon}(x, t)\right] \varphi(x, t) d x d t=0, \\
& \lim _{\varepsilon \rightarrow+0} \int_{0}^{\infty} \int L_{3}\left[u_{\varepsilon}(x, t), v_{\varepsilon}(x, t), w_{\varepsilon}(x, t)\right] \varphi(x, t) d x d t=0 .
\end{aligned}
$$

According to the weak asymptotic method, to prove that the limiting distributions 27) constitute a solution of the corresponding Cauchy problem, we multiply the first two relations in (25) (or the first three relations in (26)) by a test function $\varphi(x, t) \in$ 
$\mathcal{D}(\mathbb{R} \times[0, \infty))$, integrate these relations by parts and then pass to the limit as $\varepsilon \rightarrow+0$. Next, we must prove that the pair of distributions $(u, v)$ in Definition 2.1 (or the triple of distributions $(u, v, w)$ in Definition 3.1) satisfies the integral identities (11) (or (20)), correspondingly.

In [1, 4, 5], 14 - 15, by using the weak asymptotics method, the Cauchy problems (2), (5); (3), (5); 4), (5); admitting $\delta$-shocks were solved. In [13, [16, by using the weak asymptotics method, for the case $f(u)=u^{2}$ the Cauchy problem (7), (8) admitting $\delta^{\prime}$-shocks was solved.

\section{Algebraic aspects of singular solutions}

5.1. The problem of multiplication of distributions. As was already mentioned above, to introduce singular solutions to a nonlinear system, we need to solve the problem of multiplication of distributions. One of the approaches to this problem is the theory of nonconservative product [10, [2]. This approach generalizes the concept of Volpert's averaged superposition. In [2, a general framework for the nonconservative product

$$
g(u) \frac{d u}{d x}
$$

was introduced, where $g: \mathbb{R}^{n} \rightarrow \mathbb{R}^{n}$ is a locally bounded Borel function, and $u:(a, b) \rightarrow$ $\mathbb{R}^{n}$ is a discontinuous function of bounded variation. In [10, by using this approach, a $\delta$-shock in system (2) for the case $g(u)=f^{\prime}(u)$ was constructed. Another approach is the well-known Colombeau theory. Applications of this approach to nonlinear equations are described in many papers and books (see [1], [12]).

5.2. Singularities of flux-functions. It seems natural to introduce the product of the Heaviside function and the delta function as the weak limit of the product of their regularizations. In order to construct the regularization $f(x, \varepsilon)$ of a distribution $f \in \mathcal{D}^{\prime}(\mathbb{R})$ we use the representation

$$
f(x, \varepsilon)=f(x) * \frac{1}{\varepsilon} \omega\left(\frac{x}{\varepsilon}\right), \quad \varepsilon>0,
$$

where $*$ is the convolution, and the mollifier $\omega$ has the following properties: (a) $\omega \in$ $C^{\infty}(\mathbb{R})$, (b) $\omega(\eta)$ has a compact support or decreases sufficiently rapidly, as $|\eta| \rightarrow \infty$, (c) $\int \omega(\eta) d \eta=1, \quad(\mathrm{~d}) \omega(\eta) \geq 0, \quad(\mathrm{e}) \omega(-\eta)=\omega(\eta)$. It is known that

$$
\lim _{\varepsilon \rightarrow+0}\langle f(\cdot, \varepsilon), \varphi(\cdot)\rangle=\langle f(\cdot), \varphi(\cdot)\rangle \quad \text { for all } \varphi(x) \in \mathcal{D}(\mathbb{R}) .
$$

According to $29, \delta(x, \varepsilon)=\frac{1}{\varepsilon} \omega_{\delta}\left(\frac{x}{\varepsilon}\right)$ is a regularization of the delta function, and

$$
H(x, \varepsilon)=\omega_{0}\left(\frac{x}{\varepsilon}\right)=\int_{-\infty}^{\frac{x}{\varepsilon}} \omega(\eta) d \eta,
$$

is a regularization of the Heaviside function, $x \in \mathbb{R}$, where $\omega_{0}(z) \in C^{\infty}(\mathbb{R})$, and $\lim _{z \rightarrow+\infty} \omega_{0}(z)=1, \lim _{z \rightarrow-\infty} \omega_{0}(z)=0$. Here the mollifiers $\omega_{\delta}, \omega$ have properties (a)(e). Since the function $\omega_{\delta}(\eta) \omega_{0}(\eta)$ decreases sufficiently rapidly as $|\eta| \rightarrow \infty$, we have for $\psi(x) \in \mathcal{D}(\mathbb{R})$

$$
\left\langle\frac{1}{\varepsilon} \omega_{\delta}\left(\frac{\cdot}{\varepsilon}\right) \omega_{0}\left(\frac{\cdot}{\varepsilon}\right), \psi(\cdot)\right\rangle=\int \omega_{\delta}(\eta) \omega_{0}(\eta) \psi(\varepsilon \eta) d \eta=A \psi(0)+O(\varepsilon), \quad \varepsilon \rightarrow+0 .
$$


Thus one can define the product as

$$
\overbrace{H(x) \delta(x)}^{\text {def }} \lim _{\varepsilon \rightarrow+0} H(x, \varepsilon) \delta(x, \varepsilon)=A \delta(x),
$$

where $A=A\left(\omega_{0}, \omega_{\delta}\right)=\int \omega_{0}(\eta) \omega_{\delta}(\eta) d \eta$. The product defined in this way depends on the mollifiers $\omega, \omega_{\delta}$, i.e., on the regularizations of the distributions $H(x), \delta(x)$.

In a similar way, we can introduce the singular superpositions for flux-functions $F(u, v), G(u, v)$ associated with system (1). Let $u(x, t, \varepsilon), v(x, t, \varepsilon)$ be the regularizations of the distributions $u(x, t), v(x, t)$ in $(6)$. Then we define singular superpositions by the formulas:

$$
\begin{aligned}
& \overbrace{F(u, v)}^{\text {def }}=\lim _{\varepsilon \rightarrow+0} F(u(x, t, \varepsilon), v(x, t, \varepsilon)), \\
& \overbrace{G(u, v)} \stackrel{\text { def }}{=} \lim _{\varepsilon \rightarrow+0} G(u(x, t, \varepsilon), v(x, t, \varepsilon)),
\end{aligned}
$$

if the limits exist in the weak sense. Similarly we can introduce singular superpositions

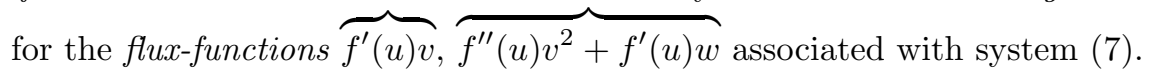

It is easy to see that these singular superpositions either depend on the regularizations of the distributions $H, \delta, \delta^{\prime}$ or do not exist in the sense of distributions (see [1], 44, [5], [13], [15]). This fact implies that the above introduced singular superpositions are not unique. However, in the context of constructing $\delta$ - and $\delta^{\prime}$-shock solutions to the Cauchy problems we can define explicit formulas for the "right" unique singular superpositions. They are unique Schwartz distributions.

THEOREm 5.1. Let $(u, v)$ be a $\delta$-shock type solution (6) to the Cauchy problem (1), (5), and let $\left(u_{\varepsilon}, v_{\varepsilon}\right)$ be its weak asymptotic solution (see Definition 4.1]. Then for $t \in[0, T$ ) we can define the explicit formulas for the "right" singular superpositions:

$$
\begin{aligned}
F(u, v) & \stackrel{\text { def }}{=} \lim _{\varepsilon \rightarrow+0} F\left(u_{\varepsilon}, v_{\varepsilon}\right)=F\left(u_{+}, v_{+}\right)+[F(u, v)] H(-x+\phi(t)), \\
G(u, v) & \stackrel{\text { def }}{=} \lim _{\varepsilon \rightarrow+0} G\left(u_{\varepsilon}, v_{\varepsilon}\right) \\
& =G\left(u_{+}, v_{+}\right)+[G(u, v)] H(-x+\phi(t))+e(t) \dot{\phi}(t) \delta(-x+\phi(t)),
\end{aligned}
$$

where the limits are understood in the weak sense, $\dot{\phi}(t), \dot{e}(t)$ are given by 16 .

Proof. Let $\left(u_{\varepsilon}(x, t), v_{\varepsilon}(x, t)\right)$ be a weak asymptotic solution to the Cauchy problem (1), (5). In view of (25), we have

$$
u_{\varepsilon t}+\left(F\left(u_{\varepsilon}, v_{\varepsilon}\right)\right)_{x}=o_{\mathcal{D}^{\prime}}(1), \quad v_{\varepsilon t}+\left(G\left(u_{\varepsilon}, v_{\varepsilon}\right)\right)_{x}=o_{\mathcal{D}^{\prime}}(1), \quad \varepsilon \rightarrow+0 .
$$

Moreover, relations 27 hold, where $(u(x, t), v(x, t))$ is a $\delta$-shock wave type solution (6) of the Cauchy problem (1), (5). By definition, the "right" singular superpositions are defined as the weak limits

$$
F(u, v) \stackrel{\text { def }}{=} \lim _{\varepsilon \rightarrow+0} F\left(u_{\varepsilon}, v_{\varepsilon}\right), \quad G(u, v) \stackrel{\text { def }}{=} \lim _{\varepsilon \rightarrow+0} G\left(u_{\varepsilon}, v_{\varepsilon}\right),
$$

where the pair of distributions $(u, v)$ is given by (6). Next, according to $(32)$, (27), we 
have

$$
\begin{aligned}
& \lim _{\varepsilon \rightarrow+0}\left\langle u_{\varepsilon t}, \varphi\right\rangle+\lim _{\varepsilon \rightarrow+0}\left\langle\left(F\left(u_{\varepsilon}, v_{\varepsilon}\right)\right)_{x}, \varphi\right\rangle=\lim _{\varepsilon \rightarrow+0}\left\langle o_{\mathcal{D}^{\prime}}(1), \varphi\right\rangle=0, \\
& \lim _{\varepsilon \rightarrow+0}\left\langle v_{\varepsilon t}, \varphi\right\rangle+\lim _{\varepsilon \rightarrow+0}\left\langle\left(G\left(u_{\varepsilon}, v_{\varepsilon}\right)\right)_{x}, \varphi\right\rangle=\lim _{\varepsilon \rightarrow+0}\left\langle o_{\mathcal{D}^{\prime}}(1), \varphi\right\rangle=0,
\end{aligned}
$$

for all $\varphi \in \mathcal{D}(\mathbb{R} \times[0, \infty))$. Thus $(34),(33)$ for all $\varphi(x, t) \in \mathcal{D}(\mathbb{R} \times[0, \infty))$ imply

$$
\begin{aligned}
& \left\langle(F(u, v))_{x}, \varphi\right\rangle=\lim _{\varepsilon \rightarrow+0}\left\langle\left(F\left(u_{\varepsilon}, v_{\varepsilon}\right)\right)_{x}, \varphi\right\rangle=-\left\langle u_{t}, \varphi\right\rangle, \\
& \left\langle(G(u, v))_{x}, \varphi\right\rangle=\lim _{\varepsilon \rightarrow+0}\left\langle\left(G\left(u_{\varepsilon}, v_{\varepsilon}\right)\right)_{x}, \varphi\right\rangle=-\left\langle v_{t}, \varphi\right\rangle .
\end{aligned}
$$

Since $u, v$ are distributions, the $(F(u, v))_{x},(G(u, v))_{x}$ are distributions as well.

Using (35) and (6), we obtain in the weak sense

$$
\begin{aligned}
(F(u, v))_{x} & =-u_{t}=-\left(u_{+}+[u] H(-x+\phi(t))\right)_{t} \\
& =-u_{+t}-\left[u_{t}\right] H(-x+\phi(t))-[u] \dot{\phi}(t) \delta(-x+\phi(t))
\end{aligned}
$$

and

$$
\begin{aligned}
(G(u, v))_{x}= & -v_{t}=-\left(v_{+}+[v] H(-x+\phi(t))+e(t) \delta(-x+\phi(t))\right)_{t} \\
= & -v_{+t}-\left[v_{t}\right] H(-x+\phi(t)) \\
& -([v] \dot{\phi}(t)+\dot{e}(t)) \delta(-x+\phi(t))-e(t) \dot{\phi}(t) \delta^{\prime}(-x+\phi(t)) .
\end{aligned}
$$

Taking into account that for $\pm x> \pm \phi(t)$ we have

$$
u_{ \pm t}+\left(F\left(u_{ \pm}, v_{ \pm}\right)\right)_{x}=0, v_{ \pm t}+\left(G\left(u_{ \pm}, v_{ \pm}\right)\right)_{x}=0
$$

and substituting the last relations into (36), (37), we derive

$$
\begin{aligned}
(F(u, v))_{x}= & \left(F\left(u_{+}, v_{+}\right)\right)_{x}+([F(u, v)] H(-x+\phi(t)))_{x} \\
& +([F(u, v)]-[u] \dot{\phi}(t)) \delta(-x+\phi(t))
\end{aligned}
$$

and

$$
\begin{aligned}
(G(u, v))_{x}= & \left(G\left(u_{+}, v_{+}\right)\right)_{x}+([G(u, v)] H(-x+\phi(t)))_{x} \\
& +([G(u, v)]-[v] \dot{\phi}(t)-\dot{e}(t)) \delta(-x+\phi(t))-e(t) \dot{\phi}(t) \delta^{\prime}(-x+\phi(t)) .
\end{aligned}
$$

Integrating the last relations with respect to $x$, we have

$$
\begin{aligned}
F(u, v)= & F\left(u_{+}, v_{+}\right)+[F(u, v)] H(-x+\phi(t)) \\
& -\left.([F(u, v)]-[u] \dot{\phi}(t))\right|_{x=\phi(t)} H(-x+\phi(t))+C_{1}(t)
\end{aligned}
$$

and

$$
\begin{aligned}
G(u, v)= & G\left(u_{+}, v_{+}\right)+[G(u, v)] H(-x+\phi(t)) \\
& -\left.([G(u, v)]-[v] \dot{\phi}(t)-\dot{e}(t))\right|_{x=\phi(t)} H(-x+\phi(t)) \\
& +e(t) \dot{\phi}(t) \delta(-x+\phi(t))+C_{2}(t),
\end{aligned}
$$

where $C_{1}(t), C_{2}(t)$ are functions. Taking into account that for $\pm x> \pm \phi(t)$

$$
\lim _{\varepsilon \rightarrow+0} F\left(u_{\varepsilon}(x, t), v_{\varepsilon}(x, t)\right)=F\left(u_{ \pm}, v_{ \pm}\right), \quad \lim _{\varepsilon \rightarrow+0} G\left(u_{\varepsilon}(x, t), v_{\varepsilon}(x, t)\right)=G\left(u_{ \pm}, v_{ \pm}\right),
$$

and using the Rankine-Hugoniot conditions for the $\delta$-shocks (16), we conclude that (38), (39) imply $C_{1}(t)=C_{2}(t)=0$ and the fact that relations 30 and (31) hold. 
TheOREm 5.2. Let $(u, v, w)$ be a $\delta^{\prime}$-shock type solution (9) of the Cauchy problem (7), (8), and let $\left(u_{\varepsilon}, v_{\varepsilon}, w_{\varepsilon}\right)$ be its weak asymptotic solution (see Definition 4.2). Then for $t \in[0, T)$ we can define the explicit formulas for the "right" singular superpositions:

$$
\begin{aligned}
& f(u) \stackrel{\text { def }}{=} \lim _{\varepsilon \rightarrow+0} f\left(u_{\varepsilon}\right)=f\left(u_{+}\right)+[f(u)] H(-x+\phi(t)), \\
& f^{\prime}(u) v \stackrel{\text { def }}{=} \lim _{\varepsilon \rightarrow+0}\left(f^{\prime}\left(u_{\varepsilon}\right) v_{\varepsilon}\right) \\
&=f^{\prime}\left(u_{+}\right) v_{+}+\left[f^{\prime}(u) v\right] H(-x+\phi(t))+e(t) \dot{\phi}(t) \delta(-x+\phi(t)), \\
& f^{\prime \prime}(u) v^{2}+f^{\prime}(u) w \stackrel{\text { def }}{=} \lim _{\varepsilon \rightarrow+0}\left(f^{\prime \prime}\left(u_{\varepsilon}\right) v_{\varepsilon}^{2}+f^{\prime}\left(u_{\varepsilon}\right) w_{\varepsilon}\right) \\
&=f^{\prime \prime}\left(u_{+}\right) v_{+}^{2}+f^{\prime}\left(u_{+}\right) w_{+}+\left[f^{\prime \prime}(u) v^{2}+f^{\prime}(u) w\right] H(-x+\phi(t)) \\
& \\
&+(g(t) \dot{\phi}(t)+h(t)) \delta(-x+\phi(t))+h(t) \dot{\phi}(t) \delta^{\prime}(-x+\phi(t)),
\end{aligned}
$$

where the limits are understood in the weak sense, $\dot{\phi}(t), \dot{e}(t), \dot{g}(t), \dot{h}(t)$ are given by (21)-24).

Proof. Let $\left(u_{\varepsilon}(x, t), v_{\varepsilon}(x, t)\right)$ be a weak asymptotic solution of the Cauchy problem (7), (8). In view of (25), we have

$$
\begin{aligned}
u_{\varepsilon t}+\left(f\left(u_{\varepsilon}\right)\right)_{x} & =o_{\mathcal{D}^{\prime}}(1), \\
v_{\varepsilon t}+\left(f^{\prime}\left(u_{\varepsilon}\right) v_{\varepsilon}\right)_{x} & =o_{\mathcal{D}^{\prime}}(1), \\
w_{\varepsilon t}+\left(f^{\prime \prime}\left(u_{\varepsilon}\right) v_{\varepsilon}^{2}+f^{\prime}\left(u_{\varepsilon}\right) w_{\varepsilon}\right)_{x} & =o_{\mathcal{D}^{\prime}}(1), \quad \varepsilon \rightarrow+0 .
\end{aligned}
$$

Moreover, relations (27) hold, where $(u(x, t), v(x, t), w(x, t))$ is a $\delta^{\prime}$-shock wave type solution (9) of the Cauchy problem (7), (8). By Theorem 5.1, relations (40, (41) hold. Just as above, using the third equation from (43) and the third relation from (9), we obtain in the weak sense

$$
\begin{aligned}
\left(f^{\prime \prime}(u) v^{2}+f^{\prime}(u) w\right)_{x}= & \lim _{\varepsilon \rightarrow+0}\left(f^{\prime \prime}\left(u_{\varepsilon}\right) v_{\varepsilon}^{2}+f^{\prime}\left(u_{\varepsilon}\right) w_{\varepsilon}\right)_{x}=-\lim _{\varepsilon \rightarrow+0} w_{\varepsilon t}=-w_{t} \\
= & -\left(\left(w_{+}\right)_{t}+\left[w_{t}\right] H(-x+\phi(t))+[w] \dot{\phi}(t) \delta(-x+\phi(t))\right. \\
& +\dot{g}(t) \delta(-x+\phi(t))+g(t) \dot{\phi}(t) \delta^{\prime}(-x+\phi(t)) \\
& \left.+\dot{h}(t) \delta^{\prime}(-x+\phi(t))+h(t) \dot{\phi}(t) \delta^{\prime \prime}(-x+\phi(t))\right) .
\end{aligned}
$$

Taking into account that $w_{ \pm t}+\left(f^{\prime \prime}\left(u_{ \pm}\right) v_{ \pm}^{2}+f^{\prime}\left(u_{ \pm}\right) w_{ \pm}\right)_{x}=0$, for $\pm x> \pm \phi(t)$, and substituting the latter relation into 444, we obtain

$$
\begin{aligned}
\left(f^{\prime \prime}(u) v^{2}+f^{\prime}(u) w\right)_{x}= & \left(f^{\prime \prime}\left(u_{+}\right) v_{+}^{2}+f^{\prime}\left(u_{+}\right) w_{+}\right)_{x}+\left(\left[f^{\prime \prime}(u) v^{2}+f^{\prime}(u) w\right] H(-x+\phi(t))\right)_{x} \\
& +\left(\left[f^{\prime \prime}(u) v^{2}+f^{\prime}(u) w\right]-[w] \dot{\phi}(t)-\dot{g}(t)\right) \delta(-x+\phi(t)) \\
& -(g(t) \dot{\phi}(t)+\dot{h}(t)) \delta^{\prime}(-x+\phi(t))-h(t) \dot{\phi}(t) \delta^{\prime \prime}(-x+\phi(t)) .
\end{aligned}
$$

Integrating the last relations with respect to $x$, we have

$$
\begin{aligned}
f^{\prime \prime}(u) v^{2}+f^{\prime}(u) w= & f^{\prime \prime}\left(u_{+}\right) v_{+}^{2}+f^{\prime}\left(u_{+}\right) w_{+}+\left[f^{\prime \prime}(u) v^{2}+f^{\prime}(u) w\right] H(-x+\phi(t)) \\
& -\left.\left(\left[f^{\prime \prime}(u) v^{2}+f^{\prime}(u) w\right]-[w] \dot{\phi}(t)-\dot{g}(t)\right)\right|_{x=\phi(t)} H(-x+\phi(t)) \\
& +(g(t) \dot{\phi}(t)+\dot{h}(t)) \delta(-x+\phi(t))+h(t) \dot{\phi}(t) \delta^{\prime}(-x+\phi(t))+C(t),
\end{aligned}
$$

where $C(t)$ is a function. Since according to $(9), \lim _{\varepsilon \rightarrow+0}\left(f^{\prime \prime}\left(u_{\varepsilon}\right) v_{\varepsilon}^{2}+f^{\prime}\left(u_{\varepsilon}\right) w_{\varepsilon}\right)=$ 
$f^{\prime \prime}\left(u_{ \pm}\right) v_{ \pm}^{2}+f^{\prime}\left(u_{ \pm}\right) w_{ \pm}$for $\pm x> \pm \phi(t)$, we conclude that 45 implies $C(t)=0$ and the fact that relation 42 holds.

5.3. Two significant examples. In order to illustrate the "strange" and specific properties of the "right" singular superpositions, we consider two particular cases of Theorem 5.1 .

(a) In [4, [5], a $\delta$-shock wave type solution (6) of the Cauchy problem (2), (5) was constructed. According to the formulas (30), 31), for this Cauchy problem we have:

$$
\begin{gathered}
f(u)=f\left(u_{+}\right)+[f(u)] H(-x+\phi(t)), \\
v g(u)=v_{+} g\left(u_{+}\right)+[v g(u)] H(-x+\phi(t))+e(t) \frac{[f(u)]}{[u]} \delta(-x+\phi(t)),
\end{gathered}
$$

where the distributions $u, v$ are given by (6).

(b) The Cauchy problems (3), (5) and (4), (5) for the Keyfitz-Kranzer system and its generalization were solved in [14, [1]. According to the formulas $(30),(31)$, for the Cauchy problem (4), (5) we have:

$$
\begin{gathered}
f(u(x, t))-v(x, t)=f\left(u_{+}\right)-v_{+}+[f(u)-v] H(-x+\phi(t)), \\
g(u(x, t))=g\left(u_{+}\right)+[g(u)] H(-x+\phi(t))+e(t) \frac{[f(u)-v]}{[u]} \delta(-x+\phi(t)),
\end{gathered}
$$

where $u, v$ are given by (6). For the Keyfitz-Kranzer system (3) formulas (48), 49) imply

$$
\begin{gathered}
u^{2}-v=u_{+}^{2}-v_{+}+\left[u^{2}-v\right] H(-x+\phi(t)), \\
\frac{1}{3} u^{3}-u=\frac{1}{3} u_{+}^{3}-u_{+}+\left[\frac{1}{3} u^{3}-u\right] H(-x+\phi(t))+e(t) \frac{\left[u^{2}-v\right]}{[u]} \delta(-x+\phi(t)) .
\end{gathered}
$$

Note that the unique "right" singular superpositions (46), 47) are essentially different from the unique "right" singular superpositions (48), (49) and (50), (51). The main distinction between them is the following.

Taking into account that $H(x) \cdot H(x)=H(x)$, one can see that in fact, by (47), the unique "right" product of the step function and the delta function is defined by:

$e(t) \delta(-x+\phi(t)) u(x, t)=e(t) \delta(-x+\phi(t)) \cdot\left\{\begin{array}{ll}u_{-}, & x<\phi(t), \\ u_{+}, & x>\phi(t),\end{array}=e(t) \frac{[f(u)]}{[u]} \delta(-x+\phi(t))\right.$.

In the case of the Keyfitz-Kranzer system (3) and its generalization (4), formulas (48), 49) and (50), (51) do not define (!) the product of the Heaviside function and the $\delta$-function. Moreover, although according to (6), $u(x, t)$ does not depend on the terms $e(t) \delta(-x+\phi(t))$ and $\left.[v(x, t)]\right|_{x=\phi(t)}$, the "right" singular superposition $g(u(x, t))$ (or $\frac{1}{3} u^{3}-$ $u$ ) determined by (49) (or (51)) does depend (!) on these terms. Thus one can say that the term $e(t) \frac{[f(u)-v]}{[u]} \delta(-x+\phi(t))$ or $e(t) \frac{\left[u^{2}-v\right]}{[u]} \delta(-x+\phi(t))$ "appears from nothing". Similarly, the left-hand sides of relations (48) and (50) depend on the term $e(t) \delta(-x+\phi(t))$ while 
the right-hand sides in (48) and (50) are independent of this term. Nevertheless, in the context of solving the Cauchy problem, the flux-function is determined uniquely.

Since the nonlinear terms in systems (3), 47), and (7) can not be reduced to terms of the form (28), it is impossible to construct $\delta$-shocks for systems (3), (4) and $\delta^{\prime}$-shocks for system (7) by using the nonconservative product [10], [2].

In [6], the system of conservation laws

$$
u_{t}+\left(\frac{u^{2}}{2}\right)_{x}=0, \quad v_{t}+(u v)_{x}=0, \quad w_{t}+\left(\frac{v^{2}}{2}+u w\right)_{x}=0
$$

was studied. This system has repeated eigenvalues. As stated in [6], system (52) cannot be solved in the classical distributional sense, therefore it is necessary to define a generalized solution in the Colombeau sense. In [6] this is motivated by the following arguments: if $v_{-}+v_{+} \neq 0$ then the $v$ component contains a $\delta$ measure along $x=0$. Though the product $u v$ does not make sense in the classical theory of distributions, it can be defined in the sense of the approach [2, but $v^{2}$ contains a square of $\delta$ measure and thus cannot be defined in this sense. It is clear that by the change of variables $u \rightarrow 2 u, v \rightarrow 2 v$, $w \rightarrow w$ system 52 can be transformed into system (7), where $f(u)=u^{2}$. Thus, contrary to the assertion from the paper [6], according to [13], system (52) admits a $\delta^{\prime}$-shock wave type solution. This solution considered in the sense of Definition 3.1 is a distributional solution. Thus we can see that the problem of introducing singular solutions to system (52) is reduced to the problem of the "right" definition of singular solutions. In the above-mentioned case a generalized solution of system (52) is represented by Schwartz distributions but not Colombeau generalized functions.

Due to the above facts, it is important to describe systems of conservation laws whose singular solutions can be defined only in terms of the Colombeau generalized functions (while they can not be defined in the sense of Schwartz distributions). It is important also to develop the theory of flux-function singularities of systems of conservation laws.

Acknowledgments. Research of the author supported by DFG Project 436 RUS 113/823, DFG Project 436 RUS 113/895, and Grant 05-01-00912 of Russian Foundation for Basic Research.

\section{References}

[1] S. Albeverio and V. M. Shelkovich, On the delta-shock front problem, in: Analytical Approaches to Multidimensional Balance Laws, Ch. 2, O. S. Rozanova (ed.), Nova Science Publishers, 2005, 45-88.

[2] G. Dal Maso, P. G. Le Floch and F. Murat, Definition and weak stability of nonconservative products, J. Math. Pures Appl. 74 (1995), 483-548.

[3] V. G. Danilov, G. A. Omel'yanov and V. M. Shelkovich, Weak Asymptotics Method and Interaction of Nonlinear Waves, in: M. Karasev (ed.), Asymptotic Methods for Wave and Quantum Problems, Amer. Math. Soc. Transl. Ser. 208 (2003), 33-165.

[4] V. G. Danilov and V. M. Shelkovich, Delta-shock wave type solution of hyperbolic systems of conservation laws, Quart. Appl. Math. 63 (2005), 401-427. 
[5] V. G. Danilov and V. M. Shelkovich, Dynamics of propagation and interaction of deltashock waves in conservation law systems, J. Differential Equations 211 (2005), 333-381.

[6] K. T. Joseph, Explicit generalized solutions to a system of conservation laws, Proc. Indian Acad. Sci. Math. Sci. 109 (1999), 401-409.

[7] R. P. Kanwal, Generalized Functions: Theory and Technique, Birkhäuser, Boston, 1998.

[8] B. L. Keyfitz and H. C. Kranzer, Spaces of weighted measures for conservation laws with singular shock solutions, Journal of Differential Equations 118 (1995), 420-451.

[9] A. Yu. Khrennikov and V. M. Shelkovich, On the algebraic aspect of singular solutions to conservation laws systems, in: Mathematical Modelling of Wave Phenomena (Växjö, 2005), B. Nilsson and L. Fishman (eds.), AIP Conference Proc. 834, 2006, 206-213.

[10] P. Le Floch, An existence and uniqueness result for two nonstrictly hyperbolic systems, in: Nonlinear Evolution Equations That Change Type, IMA Vol. Math. Appl. 27, SpringerVerlag, 1990, 126-138.

[11] M. Oberguggenberger, Multiplication of Distributions and Applications to Partial Differential Equations, Pitman Research Notes in Mathematics 259, Longman, Harlow, 1992.

[12] M. Oberguggenberger, Generalized functions in nonlinear models - a survey, Nonlinear Anal. 47 (2001), 5029-5040.

[13] E. Yu. Panov and V. M. Shelkovich, $\delta^{\prime}$-Shock waves as a new type of solutions to systems of conservation laws, J. Differential Equations 228 (2006), 49-86.

[14] V. M. Shelkovich, Delta-shock waves of a class of hyperbolic systems of conservation laws, in: A. Abramian et al. (eds.), Patterns and Waves, AkademPrint, St. Petersburg, 2003, $155-168$.

[15] V. M. Shelkovich, Delta-shocks, the Rankine-Hugoniot conditions, and singular superposition of distributions, in: Proc. Int. Seminar Days on Difraction '2004, St. Petersburg, 2004, 175-196.

[16] V. M. Shelkovich, The Riemann problem admitting $\delta$-, $\delta^{\prime}$-shocks, and vacuum states (the vanishing viscosity approach), J. Differential Equations 231 (2006), 459-500. 